\title{
L'Acadie de l'ultime déportation
}

\author{
Hans R. Runte
}

\section{Université Dalhousie}

$\prod$ fut un temps où la littérature créait l'Acadie et l'Acadie était sa littérature, où écrire équivalait à écrire l'Acadie, et où l'indéfinissable Acadie se concrétisait enfin, et peut-être pour la premiére fois, dans la littéréalité de ses textes. Ce temps des bouleversantes années 70 appartient aujourd'hui, au début des années 90 , à l'histoire (déjà !), car, l'ayant fondée au cours de la première décennie, la littérature, en se transformant, a radicalement transformé l'Acadie pendant la deuxième.

Au commencement, au seuil des années 70 , était le cri, le cri étouffé de la Sagouine ${ }^{1}$ et le cri de terre de Raymond LeBlanc ${ }^{2}$ qui, après seize ans de silence, sous-titre " 20 ans de poésie » un poème paru en 1988 dans Chants d'amour et d'espoir. Au commencement, y reconnaît-il, était la " poésie termite » aux dents rongeantes, la " poésie chauve-souris au radar sûr, » la " poésie sangsue, » la " poésie porc-épic, » puis, au son du « métal qui déchire jusqu'au coeur du silence, " la poésie devient chevreuil et s'écroule, devient hibou et se réfugie dans la nuit, devient taupe enfin, ancienne "visionnaire" désormais aveugle dans «ses galeries souterraines. $»^{3}$ Dix ans plustard, Gérald Leblanc ditplus directement : " L'Acadie chaude s'écarte d'un discours linéaire ${ }^{4}$ pour se lancer, comme le fera France Daigle dans ses romans, sur le chemin d'" un langage autre qu' une linéarité maladive de la culture occidentale. $»^{5}$

Quel était donc ce discours qui devait mener droit au but? Il partait $d^{\prime}$ un sentiment profond d'impuissance :

quand il faudra repenser

la circonférence illicite de notre massacre

nous retomberons

perplexes

en état de bétise aux couleurs de crânes

[...]

rien que pour nous miroiter 
[...]

dans les ruisseaux

de nos honyeuses masturbations, ${ }^{6}$

pour mener à la révolte :

... nous ne voulons plus être folkloriques, $[\ldots]$ nous ne voulons plus qu'on ait pitié de nous, $[. .$.$] nous ne$ voulons plus être la capitale du wellfare, $[\ldots]$ nous ne voulons plus être les assoiffés de la bonne piastre, [...] nous ne voulons plus être en stand by dans un cirque ou un studio de télévision ou un zoo, ${ }^{7}$

et c'est pourquoi

Déjà

Les femmes de la Révolte montrent leur cul au député

Le quai brûle à Caraquet

On occupe l'University

On envoie poliment chier le Délégué

Suivre l'exemple et crier. ${ }^{8}$

Le parcours des années 70 était fléché de panneaux affichant un seul mot : Acadie. En 1972 Raymond LeBlanc y inscrivit:

Je suis acadien

Ce qui signifie

Multiplié fourré dispersé acheté aliéné vendu révolté

Homme déchiré vers l'avenir ;

en 1973, Guy Arsenault écrivit :

J'ai faim d'Acadie

et j'ai soif de Parole $;{ }^{10}$

en 1975, Calixte Duguay chanta : 
Dire mon Acadie

[...]

Comme on dira un jour peut-être

Mon Kébec

Et sentir dans ses entrailles

Palpiter

Comme une envie de pain de pays

Et des frissons d'appartenance

[...]

Cela se dit avoir un pays ;1

et en 1976, Herménégilde Chiasson laissa cet appel :

Nous les nommerons Acadie ce mot longtemps lâché, longtemps pointé, défiguré, ce mot séché entre les pages, $[\ldots]$ cette colère qui brûle les yeux au feu de la honte $[\ldots] .^{12}$

$\mathrm{Au}$ bout de ce chemin Léonard Forest entrevoyait sa «sur allégorique » nommée Acadie,

toi

[...] mon pays

j'y lancerai le troupeau de mes désirs

enfin rapatriés

j'en nommerai tous les vallons et

toutes les pointes

j'en connaîtrai l'unique espoir

je l'épouserai

toi

tu es mon pays

j'y naîtrai. ${ }^{\text {?3 }}$

Avec plus de véhémence, Raymond LeBlanc affirmait :

Voici l'heure des hommes éveillés

[...]

Voici la marche vers l'avenir

[...]

Voici l'heure de l'histoire voulue 
Pour changer la misère des esclaves

Dans la réalité des hommes nouveaux et libres

Cette heure désormais nous appartient. ${ }^{14}$

Ainsi le désir de dire éclata, la poésie arriva en vie, en bourrasque, à $l^{\prime}$ aventure de liberté $;^{15}$ mais c'est «à tangage réglé à l'espace des mots précis $»^{16}$ que la poésie, cette $"$ difficile tendresse, ${ }^{17}$ fonda $1^{\prime}$ Acadie et l'Acadie était poésie, fusion de vent et de marbre, ${ }^{18}$ vision transformée en promesse lucide. ${ }^{19}$

L'espace d'une décennie, "c'est ici le lieu du poème, " tant dans "l'herbe des collines fraîches » que dans les « murs d' une ville machine $»^{20}$ comme Moncton ; « c'est ici le lieu » aussi de la prose, des pièces enragées de Jules Boudreau et de Claude Renaud, du guide démythificateur et de l'histoire de la non-conformiste et rebelle Maria à Gélas d'Antonine Maillet, ${ }^{21} \mathrm{du}$ roman d'anticipation de Claude LeBouthillier (1977). Tous disent, " à coup[s] d'amour à force de rêves, " ${ }^{22} \mathrm{l}^{\prime}$ Acadie et son acadianité, et tous sauf Antonine Maillet seront, au début des années 80, cruellement déçus :

les illusions se dégonflent

à la vitesse de la lumière

dans le débris des années $70,{ }^{23}$

et les écrivains se retrouvent

projetés dans [leurs] mots, inutiles, comme d'autres dans leurs doutes. ${ }^{24}$

Comment, en moins de dix ans, l'Acadie a-t-elle pu s'avérer pour beaucoup « un territoire trop serré ${ }^{25}$ dont le « centre d'énergie [...] est sans point de repère, $»^{26}$ où $~ «$ il n'y a pas de noyau $»^{27}$ ? Peut-être $s^{\prime}$ agit-il du même phénomène que $V$. S. Naipaul observa jadis aux ÉtatsUnis : "The American Negro's subject is his blackness ... and once [he] has made his statement, his profitable protest, he has nothing to say. ${ }^{28}$ L'Acadie dite, l'Acadien n'a plus rien à dire. Mais il n'y a pas que cela en Acadie. Ce qui sape les assises du pays à peine rené, au sortir des années 70 , c'est aussi la nécessité d'admettre que « la trappe à homard et le bateau au bout du quai » ne pouvaient plus être « des sujets de poésie. »" $\mathrm{A}$ ce refus de la folklorisation s'ajoutent la constatation des poètes selon laquelle leur nouvelle Acadie a fini par ressembler à l'ancienne : 
l'exploitation

se porte bien chez nous

à l'université on soigne le français comme à l'hôpital, ${ }^{30}$

et la découverte que, même en anticipant, un romancier comme Claude LeBouthillier n'a pu faire mieux que de récostumer l'Acadie à venir dans les clichés et le discours du passé (1977). Enfin, l'échec des projets de pays littéraires des années 70 coïncide avec une curieuse inquiétude millénariste selon laquelle « l'époque se désintégre, » ${ }^{31}$

le monde se dissout comme les rides

au coeur de l'avalanche ${ }^{32}$

et les écrivains créent

dans le désordre de la fin d'un siècle

qui semble être le dernier. ${ }^{33}$

En réponse à cette crise de conscience, et après les innombrables dérangements acadiens, grands et petits, s'échelonnant de 1755 à Jackie Vautour $^{34}$ en passant par Joseph Des Barres ${ }^{35}$ et Louis Mailloux, ${ }^{36}$ la deuxième décennie de la littérature acadienne moderne aura vu la plus vaste et la plus radicale déportation de toutes. Déboussolés, fatigués des combats d'antan, incertains des anciennes certitudes, aussi précaires fussent-elles, désespérant des vieux espoirs, les écrivains acadiens auront expulsé l'Acadie de la littérature acadienne, auront évacué le signifié acadien et abandonné leurs bataillons de signifiants revendicateurs pour s'exiler dans la postacadianité. Tous les efforts visant à fonder l'Acadie en l'écrivant s'étant soldés par un échec, l' Acadie se trouve à jamais bannie des préoccupations de ses écrivains, donc, cesse d'exister. Après elle, quoi ?

Après elle le néant, du moins selon France Daigle qui, mieux que nul(le) autre, a compris que la disparition de l'Acadie a pour raison fondamentale et inéluctable, à l'époque postmoderne qui est la nôtre, l'impossibilité de l'écriture. Aussi ses textes non seulement parlent-ils de moins en moins de son pays, mais se taisent-ils de plus en plus, offrant des pages de plus en plus blanches où « s'instaurer dans le silence comme dans la solidité des choses. " ${ }^{37}$ 
D'autres exilés, à peine moins pessimistes, se replient sur eux-mêmes pour trouver un point $\mathrm{d}^{\prime}$ attache dans un intimisme parfois contemplatif, ${ }^{38}$ parfois hallucinatoire :

les arguments que j'ai dans

ma tête avec toi ou est-ce moi

qui me parle en monologue incessant

$[\ldots]$

d'où viennent ces voix

que je nomme parfois poésie, $^{39}$

et parfois, hélas, tout à fait banal :

l'important c'est de vivre sa vie, oser un mieux-être, ${ }^{40}$

ou

J'ai dépassé [l]e stade de révolte et de violence. [...]

Je réalise qu'il est préférable d'aimer et de s'entourer de beaux moments, que de vivre avec le poil à pic sur l'échine ! [...] [J]e veux vivre les choses telles $q^{\prime}$ 'elles $m^{\prime}$ apparaissent, telles que je les ressens [...]. J'ai besoin d'être bien, d'avoir une écriture où les choses s'équilibrent, s'harmonisent. Une écriture où il fait beau vivre ! ${ }^{41}$

Cette écriture-là, rappelons-le, ne s'appelle plus Acadie ; elle s'appelle, lorsqu'elle assume sa déportation comme le fait celle, exemplaire et poétique, de Gérald Leblanc, Amérique. Contre l'échec des années 70 , contre le repliement des désillusionnés, contre $l^{\prime}$ engouement anachronique pour le roman historique (LeBouthillier 1989), il y a " la dérive nécessaire, " ${ }^{42}$ il y a

les taxis [qui] sont là pour nous dériver

$[\ldots]$

et je suis comme Jack Kérouac. ${ }^{43}$

Poètes et romanciers (parmi ceux-ci, Jacques Savoie), dont 
la tête va et vient

[...]

sur les routes d'Acadie

sur toutes les routes de l'imaginaire

mouvance

déraciné[s] apatride[s], ${ }^{44}$

découvrent, après avoir longtemps dénoncé l'Amérique en Acadie, l'Acadie dans l'Amérique. L'Acadien sans Acadie est le beatnik " on the road, " le Noir du blues et du jazz, l'Amérindien sans terre, le consommateur de l'Amérique américaine, et sa patrie est le « continent qui $\left[l^{\prime}\right]$ écrit ${ }^{45}$ et l'écriture de l'errance, cette errance qui

[...] ouvre la porte

comme on ouvre les bras devant l'infini. ${ }^{46}$

Ainsi libérés des contraintes de l'histoire et du régionalisme, les écrivains de l'acadianité américaine (dont Guy Arsenault, selon Raymond LeBlanc, ${ }^{47}$ fut le premier poète) forgent de nouvelles allégeances et se découvrent de nouvelles appartenances. Mais le concept de l'acadianité américaine, non moins que celui de la francophonie (qui n'a jamais intéressé les Acadiens), a tout pour gober d'un trait la spécificité acadienne si péniblement, et peut-être si illusoirement, élaborée pendant les années 70 . Dire que «les livres acadiens et québécois s'inscrivent dans la bibliothèque de l'univers, » comme le fit le Québécois Claude Beausoleil, ${ }^{48}$ est plus une tape fraternelle d'encouragement qu'un constat réaliste de l'état des choses acadiennes. D'autre part, s'ouvrir à " l'immense

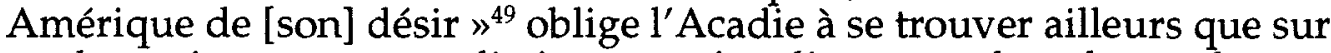
quelque rive sauve et solitaire un point d'ancrage dans le maelstrom d'un présent fragmenté, chaotique et aliénant. Au sortir des années 80 , les mots des poètes surtout ne sont que rumeurs, fantasmes, déchirures, grimaces, hallucinations, mots durs brisés au choc du continent traquant une réalité constamment mise en doute, 50 mots qui risquent d'institutionaliser

la question sans réponse

l'errance pour l'errance. ${ }^{51}$ 
Le meilleur indice que l'errant tourne en rond, le signe de «l'aboutissement d'une époque » où régnait le quotidien, $c^{\prime}$ est, selon Herménégilde Chiasson,
$[$ [...] la longue consultation des catalogues
Les répertoires de l'obsession, la cartographie
des mirages [...] les sessions abyssales
dans les cabinets du doute,
la magie intercédante des signes. ${ }^{52}$

Aussi l'aspect recenseur des recueils du chantre de l'errance, Gérald Leblanc (les disques écoutés, les livres lus, les bouteilles vidées, les cigarettes fumées), est-il susceptible de démentir le poète qui voudrait que « le monde qu['il] revendique [soit] inouii » 53 (du moins était-il plus crédible en 1974 quand il lança : »le temps est venu pour nous de crier nos poèmes à tue-tête $"^{54}$ ).

Seul Herménégilde Chiasson a proposé, pour les années 90, une manière d'errer vers un but, un mouillage possible dans la vaste américanisé acadienne (le transcendantalisme asiatique du Raymond LeBlanc de Chants d'amour et d'espoir me semble d'un apport beaucoup moins solide) :

Nous sommes venus par la mer aux Amérindiens comme des extra-terrestres [...]

$[\ldots]$

Leur monde [...] allait

disparaître dans la bande dessinée rose et mauve qu'allait devenir l'Amérique. L'Amérique allait se manifester. L'Amérique s'est manifestée.

Et nous sommes peut-être ces gens-là, descendants de

coureurs de bois $[\ldots]$

$[\ldots]$

Nous sommes les survivants de toutes les rumeurs et de

toutes les peurs imaginables.

Notre fragilité n'a d'égale que notre endurance. ${ }^{55}$ 
L'errance et la littérature seraient alors une « errance amérindienne [...] peut-être notre seule dimension possible » et qui permet de participer " à un monde spirituel, à une sorte d'extase existentielle qui consiste en une présence intense, » et de découper « dans le quotidien des moments d'une charge insoupçonnée. $)^{56}$

Voilà, pour la prochaine génération d'écrivains dits acadiens, un discours qui tente $\mathrm{d}^{\prime}$ 'établir des relations harmonieuses, [de] se mettre en accord avec la dimension spirituelle de l'univers, $\nu^{57}$ un discours non plus simplement de l'acadianité, américaine ou autre, mais de l'acadianitude.

\section{Notes}

${ }^{1}$ Maillet 1971.

${ }^{2}$ LeBlanc 1972.

${ }^{3}$ LeBlanc 1988 : 29.

${ }^{4}$ Leblanc, "La fin des années '70, » 1981 : s.p.

${ }^{5}$ Leblanc, « La fin des années '70, » 1981 : s.p.

'Landry, "Nos rues de sang froid, " $1977: 29$.

${ }^{7}$ Chiasson, "Quand je deviens patriote, » 1974 : 39.

8Jean, « R'garde m'man, j' parle, » $1982: 13$.

${ }^{9}$ LeBlanc, « Je suis acadien, » 1972: 53.

${ }^{10}$ Arsenault, Acadie Rock, $1973: 21$.

${ }^{11}$ Duguay, « Avoir un pays, " $1975: 23$ et 25.

${ }^{12}$ Chiasson, " 10 incantations pour que le pays vienne, " $1976: 43$.

${ }^{13}$ Forest, «Pour une soeur allégorique, III, » $1973: 64$.

${ }^{14}$ LeBlanc, "Projet de pays (Acadie-Québec), " $1972: 45$.

${ }^{15}$ Leblanc, « La poésie, » 1981 ss.p.

${ }^{16}$ LeBlanc, "Projet de pays (Acadie-Québec), " $1972: 45$. 
${ }^{17}$ LeBlanc, « Poésie, » 1972 : 9.

${ }^{18}$ LeBlanc, «Poésie, » 1972 : 9.

${ }^{19}$ LeBlanc, « Projet de pays (Acadie-Québec), » $1972: 45$.

${ }^{20}$ LeBlanc, « Poésie, » 1972 : 9.

${ }^{21}$ Maillet 1973a, 1973b.

${ }^{22}$ LeBlanc, «Projet de pays (Acadie-Québec), » $1972: 45$.

${ }^{23}$ Leblanc, « Graffiti bleu, » $1988: 67$.

${ }^{24}$ Chiasson, « Désir, » $1986: 27$.

${ }^{25}$ Leblanc, « September song (pour ma fête), » 1984b : 18.

${ }^{26}$ Bourque, «Paysage, » $1988: 121$.

${ }^{27}$ Dugas 1988 : 114.

${ }^{28}$ Naipaul $1988: 75$.

${ }^{29}$ Leblanc, «Visions de Rimbaud (Projet d'autobiographie), » $1986: 41$.

${ }^{30}$ Leblanc, [sans titre,] $1988: 34$.

${ }^{31}$ Leblanc, « Danser au Kacho, » $1986: 27$.

${ }^{32}$ Chiasson, "Boursouflures, " $1986: 13$.

${ }^{33}$ Leblanc, « Toujours des rêves tombent, 1, " $1988: 137$.

${ }^{34}$ Voir Leblanc 1981 : s.p., 1988 : 57.

${ }^{35}$ Voir L. Thériault $1980: 54$.

${ }^{36}$ Voir Duguay 1975 : 63-65, Duguay et Boudreau 1976.

${ }^{37}$ Daigle $1983: 139$.

${ }^{38}$ Voir, par exemple, J. Thériault 1981, 1983 ; Saint-Pierre 1986, 1990 ; Albert-Lévesque1988.

${ }^{39}$ Leblanc, « Voix, " $1988: 157$.

${ }^{40}$ Leblanc, « La fin des années '70, » 1981 : s.p. 
${ }^{41}$ Léger 1986 : 34-35.

${ }^{42}$ Leblanc, « September song (pour ma fête), » 1984b : 18.

${ }^{43}$ Leblanc, « Fast food and quick snacks, » $1988: 69$.

${ }^{44}$ Leblanc, « Toujours des rêves tombent, 8, » $1988: 144$.

${ }^{45}$ Leblanc 1984a : 141.

${ }^{46}$ Leblanc, "Toujours des rêves tombent, 8, » $1988: 144$.

${ }^{47}$ LeBlanc 1984 : 29-31.

${ }^{48}$ Beausoleil $1984: 70$.

${ }^{49}$ Leblanc, « Multipiste, » $1988: 106$.

${ }^{50}$ Beausoleil $1988: 16-17$.

${ }^{51}$ Leblanc, «L'attrait de l'extérieur, » $1988: 121$.

${ }^{52}$ Chiasson, «Injures I, » $1986: 73$.

${ }^{53}$ Leblanc, «Poème/intervention, » 1981 : s.p.

${ }^{54}$ Leblanc, « Manifeste, " $1988: 41$.

${ }^{55}$ Chiasson, « Amériques, » $1986: 53$.

${ }^{56}$ Chiasson $1988: 12-13$.

${ }^{57}$ Chiasson $1988: 13$.

\section{Références}

Albert-Lévesque, Anne. 1988. Du haut des terres : roman. Moncton : Éditions d'Acadie.

Arsenault, Guy. Acadie Rock. Moncton : Éditions d'Acadie.

Beausoleil, Claude. 1984. [Préface.] Les cent lignes de notre américanisé : actes du colloque tenu à Moncton du 14 au 16 juin 1984. Moncton : Éditions Perce-Neige. Beausoleil 1988. 1988. «Poésie d'Acadie : en version originale. » Dans Leblanc et Boudreau, Jules. 1979. Cochu et le soleil : pièce en trois actes. 2e éd. Moncton : Éditions d'Acadie.

Bourque, Paul J. 1988. «Paysage. » Dans Leblanc et Beausoleil 1988 : 121. 
Chiasson, Herménégilde. 1974. Mourir à Scoudouc. Moncton : Éditions d'Acadie. . 1976. Rapport sur l'état de mes illusions. Moncton : Éditions d'Acadie. 1986. Prophéties. Poésie. Moncton : Michel Henry Éditeur. 1988. « Pour saluer Gérald Leblanc. » Dans Leblanc 1988. Daigle, France. 1983. Sans jamais parler du vent : roman de crainte et d'espoir que la mort arrive à temps. Moncton : Éditions d'Acadie. .1984. Film d'amour et de dépendance : chef-d'oeuvre. obscur. Moncton : Éditions d'Acadie. 1985a. Histoire de la maison qui brâle : vaguement suivi d'un dernier regard sur la maison qui brûle. Moncton : Éditions d'Acadie. .1985b. Variations en $b$ et $k$ : plans, devis et contrat pour l'infrastructure d'un pont. Auteur/e 168. Montréal : La nouvelle barre du jour. . 1991. La beauté de l'affaire : fiction autobiographique à plusieurs voix sur son rapport tortueux au langage. Montréal et Moncton : La nouvelle barre du jour et Éditions d'Acadie.

Daigle, Jean. 1980. Les Acadiens des Maritimes. Moncton : Centre d'études acadiennes, Université de Moncton.

Dugas, Daniel. 1988. [Inédit sans titre]. Dans Leblanc et Beausoleil $1988: 114$.

Duguay, Calixte. 1975. Les stigmates du silence. Moncton : Éditions d'Acadie.

Duguay, Calixte, et Jules Boudreau. 1976. Louis Mailloux : spectacle musical. SaintBruno : Éditions du Kapociré KC 5304 (disque et livret).

Forest, Léonard. 1973. Saisons antérieures : poèmes. Moncton : Éditions d'Acadie. Gallant, Melvin. 1986. «Dyane de Notre-Dame : entretien avec Dyane Léger. " Éloizes $13: 33-38$.

Jean, Guy. 1982. Paroles d'Acadie et d'après. Poètes de l'Outaouais 14. Hull : Éditions Asticou.

Landry, Ulysse. 1977. Tabous aux épines de sang. Moncton : Éditions d'Acadie. Neige.

Leblanc, Gérald. 1981. Comme un otage du quotidien. Moncton : Éditions Perce. 1984a. «Post-face. » Dans Beausoleil 1984. $1984 \mathrm{~b}$. Géographie de la nuit rouge. Moncton : Éditions d'Acadie. 1986. Lieux transitoires : poèmes et textes. Poésie. Moncton : Michel Henry Editeur. d'Acadie. 1988. L'extrême frontière : poèmes 1972-1988. Moncton : Éditions

Leblanc, Gérald, et Claude Beausoleil. 1988. La poésie acadienne 1948-1988. TroisRivières : écrits des forges / Le castor astral.

LeBlanc, Raymond. 1972. Cri de terre : poèmes 1969-1971. Moncton : Éditions d'Acadie.

Éditeur.

1984. « Nos images Kodak. » Dans Beausoleil 1984. 1988. Chants d'amour et d'espoir. Poésie. Moncton : Michel Henry

LeBouthillier, Claude. 1977. L'Acadien reprend son pays : roman d'anticipation. Moncton et Granby : Éditions d'Acadie et Éditions Gaudet. 1989. Le feu du mauvais temps. Deux continents, série Best-Sellers.

Montréal : Québec/Amérique.

Léger, Dyane. 1986. Voir Gallant 1986. 
Maillet, Antonine. 1971. La Sagouine : pièce pour une femme seule. Répertoire acadien $1 / 2$. Montréal : Leméac.

1973a. L'A cadie pour quasiment rien : guide historique, touristique et humoristique d'Acadie. Les guides historiques et touristiques. Montréal : Leméac. 1973b. Mariaagélas : roman acadien. Roman acadien 3. Montréal : Leméac.

Naipaul, V. S. 1988 (1962). The Middle Passage. Harmondsworth : Penguin.

Renaud, Claude. 1978. Sacordjeu! Moncton : Éditions d'Acadie.

Saint-Pierre, Christiane. 1986. Sur les pas de la mer : contes et nouvelles. Moncton : Éditions d'Acadie. 1990. Absente pour la journée. Moncton: Éditions d'Acadie.

Savoie, Jacques. 1984. Les portes tournantes : roman. Montréal : Boréal Express. 1986. Le Récif du Prince : roman. Montréal : Boréal Express. 1988. Une histoire de coeur : roman. Montréal : Boréal Express.

Thériault, Jeannine Landry. 1981. Un soleil mauve sur la baie : roman. Moncton : Éditions d'Acadie.

1983. Le moustiquaire : roman. Moncton : Éditions d'Acadie.

Thériault, Léon. 1980. "L'Acadie, 1763-1978 : synthèse historique. " Dans J. Daigle 1980. 